\title{
Le rocouyer (Bixa orellana), une source de biocolorant pour les industries alimentaires: revue analytique
}

\author{
Ezéchiel AKAKPO ${ }^{1}$, Marius Eric BADOUSSI ${ }^{1,2}$, Claude Kouassi GNACADJA ${ }^{1}$, \\ Hermance HOUNGBO ${ }^{1}$, Alphonse DOSSOU ${ }^{1}$ et Paulin AZOKPOTA ${ }^{1^{*}}$ \\ ${ }^{1}$ Laboratoire de Formulation des Aliments et Biologie Moléculaire (LAFAB) Faculté des Sciences \\ Agronomiques, Université d'Abomey-Calavi; 03 BP 2819 Jéricho, Cotonou, Bénin. \\ ${ }^{2}$ Ecole Nationale Supérieure de Biosciences et Biotechnologies Appliquées (ENSBBA), Université Nationale \\ des Sciences, Technologies, Ingénierie et Mathématiques (UNSTIM), BP : 14 Dassa-Zoumé, Bénin. \\ "Auteur correspondant; E-mail : azokpotap@yahoo.fr; Tel: 00229 97221249
}

\section{RESUME}

Bixa orellana est une espèce végétale pourvoyeuse de biocolorant utilisé traditionnellement aussi bien en Asie, en Amérique qu'en Afrique. Cette revue vise à faire un récapitulatif des travaux antérieurs sur le colorant des graines de Bixa orellana afin de définir de nouveaux objectifs de recherche pour sa valorisation. La méthodologie adoptée est basée sur une documentation axée sur la pertinence et la qualité des articles. Une centaine d'articles scientifiques ont été exploités et les informations capitalisées font état de ce que Bixa orellana a une grande importance socioéconomique et alimentaire. Ses graines, fournissent un colorant ayant deux fractions; la norbixine (hydrosoluble) et la bixine (liposoluble), stables surtout aux températures inférieures à $180^{\circ} \mathrm{C}$ et, constituent ainsi, la seule source naturelle de bixine, un caroténoïde très utilisé dans l'industrie alimentaire comme colorant. Les potentialités nutritionnelles et thérapeutiques des graines font de l'espèce, un important patrimoine agricole capable de faire face aux défis de substitution de certains colorants de synthèse par ceux de ces graines. Son exploitation permettrait de pallier aux pathologies liées à l'utilisation des colorants de synthèse, d'améliorer le revenu agricole des populations pratiquant sa culture et d'offrir à l'espèce, une visibilité pour sa valorisation en Afrique et principalement au Bénin..

(C) 2019 International Formulae Group. All rights reserved.

Mots Clés: Bixa orellana, biocolorant, colorant de synthèse, valorisation agroalimentaire, Bénin.

\section{Annatto (Bixa orellana), a source of biocolorant for food industries: analytical review}

\begin{abstract}
Bixa orellana is a vegetable species provider of biocolorant used traditionally as well in Asia, in America as in Africa. This review mainly aims at summarizing the works done about Bixa orellana seeds dye in order to define research perspectives for the species valorization. The methodology is carried out starting from a documentation centered on the relevance of the articles. In total, some 100 scientific articles have been exploited. Capitalized informations show that Bixa orellana is of great socioeconomic and nutritional importance. Its seeds provide a dye with two fractions; norbixin (water-soluble) and bixin (fat-soluble), stable especially at temperatures below $180{ }^{\circ} \mathrm{C}$ and thus constitute the only natural source of bixin, a carotenoid
\end{abstract}


widely used in the food industry as a dye. The nutritional and therapeutic potentialities of seeds make the species an important agricultural holding capable to face the challenges of substitution of certain synthetic dyes with those of these seeds. Its exploitation would make it possible to palliate the pathologies related to the use of the synthetic dyes, to improve the agricultural income of the populations practicing its culture and to offer to the species, a visibility for its valorization in Africa, particularly in Benin.

(C) 2019 International Formulae Group. All rights reserved.

Keywords: Bixa orellana, biocolorant, synthetic dye, agri-food valorization, Benin.

\section{INTRODUCTION}

L'homme a mis des couleurs dans sa vie dès les débuts de son aventure. Il a d'abord utilisé les pigments des « terres colorées », puis ceux des fibres végétales et animales (Ben Mansour et al., 2011). Qu'ils se servent de pigments bruts, raffinés ou de synthèse, les humains ont rapidement créé des palettes que les millénaires n'ont pas réussi à effacer (Pigeaud, 2003). Il existe deux types de colorants à savoir les colorants naturels qui sont des pigments de légumes, de fruits, de graines ou d'épices et les colorants artificiels qui sont totalement de synthèse chimique (Cardon et al., 2010; Birr et Montavon, 2004). Les colorants de synthèse ont l'avantage par rapport aux colorants naturels d'avoir une durée de vie plus longue et de donner des couleurs plus intenses. Ils sont donc utilisés en plus petite quantité et sont moins onéreux que les colorants naturels sensibles à la lumière, à l'oxygène ou à l'action des bactéries (Birr et Montavon, 2004). Cependant, les colorants naturels dérivés de la flore et de la faune sont considérés comme sûrs en raison de leur nature non-toxique, non-allergique, noncancérigène et biodégradable (Bao et al., 2005; Sinha et al., 2012a). Ils ne créent aucun problème écologique à l'étape de la production et maintiennent l'équilibre écologique (Sivakumar et al., 2011; Sinha et al., 2012b). De plus, certains colorants naturels ont des propriétés fonctionnelles très bénéfiques telles que la réduction du stress oxydant et la capacité d'agir en tant qu'agent chémopréventif et réducteur des effets néphrotoxiques des agents anti-tumoraux (Agner et al., 2005; Rios et al., 2009). Au regard des problèmes de pollution environnementale et de toxicité liés à l'utilisation des colorants de synthèse (Magda, 2003), les industries alimentaires se retrouvent face au défi de la substitution des colorants de synthèse par les colorants naturels (Bao et al., 2005). A cet effet, de nombreuses recherches ont été orientées vers l'identification et la caractérisation des colorants de sources végétales telles que des légumes (Giusti et al., 2001), des fruits et des fleurs (Awika et al., 2004). Ainsi, de nombreuses espèces végétales fournissent des colorants naturels pour diverses utilisations humaines. Nous pouvons citer Sorghum Vulgaris (gaines foliaires), Tectona grandis L. (jeunes feuilles), Lawsonia inermis L. (feuilles), Indigofera tinctoria L. (tiges feuillées), et Bixa orellana L. (graines) (Sessou et al., 2013; Fagbohoun, 2014).

Bixa orellana est une plante originaire de l'Amérique tropicale et qui est de nos jours largement cultivée dans de nombreuses régions tropicales pour la production commerciale (Jansen, 2005; Renata et al., 2006). L'extrait de Bixa orellana est un colorant naturel alimentaire, qui est obtenu à partir de l'arille de ses graines. Pour la valorisation du biocolorant extrait des graines de Bixa orellana, en vue de susciter l'espoir d'une production massive notamment dans les pays en développement afin de réduire de façon substantielle les nombreuses maladies liées à l'utilisation des colorants de synthèse dans l'industrie agroalimentaire, il est important que la recherche soit orientée vers le développement des colorants naturels à travers le recensement des qualités nutritionnelles du colorant ainsi que ses différentes propriétés technologiques et ses applications.

Quelques travaux ont déjà été réalisés 
dans ce sens sans pour autant atteindre pleinement ces objectifs notamment en Inde et au Brésil (Venugopalan et al., 2011; Sangvikar et al., 2015; Prathima et al., 2016). Il importe donc de réaliser un état des lieux de ces travaux afin de donner une nouvelle orientation à la recherche sur le colorant extrait des graines de Bixa orellana. La présente revue vise principalement à faire un récapitulatif analytique des travaux antérieurs sur le colorant des graines de Bixa orellana à partir duquel de nouveaux objectifs de recherche pourraient être définis. De façon spécifique, il s'agit de collecter des données sur la production, l'importance socioéconomique et nutritionnelle de l'espèce Bixa orellana au plan international et régional; décrire les propriétés technologiques du colorant extrait des graines et définir des perspectives de recherche pour la valorisation de l'espèce.

\section{PRODUCTION ET IMPORTANCE SOCIO-ECONOMIQUE, NUTRITIONNELLE THERAPEUTIQUE DE BIXA ORELLANA Données botaniques et production de l'espèce Bixa orellana}

Le rocouyer du nom scientifique Bixa orellana est une espèce du genre Bixa et appartient à la famille des Bixaceae. En dépit de l'existence de plusieurs espèces, la plus connue est Bixa orellana L., baptisé du nom de Francisco Orellana, qui était le premier européen à diriger l'Amazonie (Silva et al., 2010). Bixa orellana est un petit arbre ou arbuste mesurant 3 à 5 mètres de hauteur, atteignant parfois une taille de 10 mètres (Figure 1) (de Araújo Vilar et al., 2014). Originaire d'Amérique du Sud, et retrouvé dans certains pays tropicaux, Bixa orellana est surtout cultivé pour ses graines à partir desquelles on extrait du biocolorant (Periyasamy et al., 2016). Le rendement en graines atteindrait 3-5 t/ha, mais au Sri Lanka on a fait état de rendements de seulement 625 $\mathrm{kg} / \mathrm{ha}$ (Jansen, 2005). Selon la même source, le rendement annuel en graines est normalement de $800-1200 \mathrm{~kg} / \mathrm{ha}$ soit $0,5-4$ kg par arbre.
La production mondiale de graines de roucou pour les usages domestiques et commerciaux a été estimée en 1990 à 10.000 tonnes (Jansen, 2005). Cette production a rapidement augmentée à 14500 tonnes (poids sec) par an en quelques années (Levy et Rivandeneira, 2000). Les principaux producteurs commerciaux de Bixa orellana sont des pays d'Amérique Latine (Brésil, Pérou, Mexique, Equateur, République Dominicaine, Honduras, Colombie, Bolivie, Guatemala et Costa Rica), d'Afrique (Kenya, Afrique du Sud, Zimbabwe, Côte d'Ivoire et Angola), et d'Asie (Inde et Sri Lanka) (Venugopalan et al., 2011). L'Amérique latine produit $60 \%$ de la production mondiale des graines de rocouyer, suivi de l'Afrique (27\%) et de l'Asie (12\%) (Giuliano et al., 2003). Le Pérou est le premier pays exportateur de graines de roucou (environ 4000 tonnes par an), le Brésil étant quant à lui le principal producteur avec près de 5000 tonnes (Jansen, 2005). En Afrique, le Kenya exporte à peu près 1500 tonnes par an de graines et d'extraits de roucou, ce qui fait de lui, le deuxième pays exportateur après le Pérou, notamment vers le Japon. Les Etats-Unis sont les plus grands importateurs des produits de rocouyer, suivi de l'Europe où la GrandeBretagne est le plus grand importateur et le Danemark est le plus grand transformateur (Foodnet, 2002).

Dans de nombreux pays d'Amérique tropicale, les graines sont récoltées à la fois sur les plantes cultivées dans les jardins familiaux et sur les plantes sauvages. L'espèce est aussi présente dans de nombreux pays africains dont le Burkina-Faso (Alexandre, 2002), le Bénin (Akoègninou et al., 2006), le Nigeria (Braide et al., 2011) et le Niger (PROVAPEG, 2015). Les Figures 2 et 3 illustrent respectivement la répartition de l'espèce dans le monde et en Afrique.

Importance socio-économique, thérapeutique et nutritionnelle de Bixa orellana

\section{Importance socio-économique}

En dehors de l'utilisation traditionnelle des graines comme condiment ou 
assaisonnant, les graines fournissent le deuxième colorant naturel d'importance économique (après le caramel) (Satyanarayana et al., 2003). Ce colorant, constitué essentiellement de bixine (70-80\%) et de Norbixine (20 - 30\%) (Aparnathi et al., 1990) est utilisé non seulement dans l'industrie textile, cosmétique et dans l'artisanat pour teindre les fibres (vannerie), les étoffes et divers masques mais aussi dans l'industrie agroalimentaire notamment dans la coloration des crèmes glacées, des produits laitiers, des jus et liqueurs, de la sauce, des gâteaux (Venugopalan et al., 2011; Fagbohoun, 2014). Les prix des graines de rocouyer ont varié de 600 à 2000 US\$ par tonne entre 1985 et 1995 (Giuliano et al., 2003 ) et l'extrait des graines avec $30 \%$ de bixine était de 30.000 US\$ par tonne en 1988 (FAO, 1995). Selon Jansen (2005) le coût moyen du marché par tonne de graines a fortement varié; entre 1984 et 1990, il est passé de 600 à 2300 US\$. Le principal marché du rocou est les Etats-Unis avec 3000 tonnes par an, suivis de l'Europe occidentale (2500 tonnes) et du Japon (1500 tonnes). En Côte d'Ivoire, depuis les années 1990, face aux difficultés du binôme Cacao-Café, la région a vu l'introduction de nouvelles cultures qui étaient porteurs d'espoir dont le roucou qui s'adapte bien aux conditions qu'offre le milieu (Mara, 1999; OCDE, 2002; Koffi, 2015). Pour Koffi (2015), la culture du roucou en Côte d'Ivoire a suscité de nombreux espoirs au sein de la population rurale puisque le kilogramme de graines de roucou était acheté à 1000 FCFA au cours des deux premières années de son introduction, période au cours de laquelle le produit était acheté par les investisseurs américains. Les prix du kilogramme pratiqués aux cours des campagnes de 2006 à 2012 ont varié de 175 FCFA à 400 FCFA plaçant ainsi le roucou comme produit rivalisant avec l'anacarde derrière le Cacao. La culture du roucou contribue avec un apport significatif au revenu des ménages producteurs contribuant ainsi à l'amélioration des conditions de vie des populations à revenus agricoles (Koffi, 2015). Les graines sont vendues après nettoyage soit pour être exportées soit pour être moulinées et vendues sur le marché local (Figure 4) ce qui génère encore plus de revenus aux revendeuses.

\section{Importance thérapeutique}

L'utilisation des plantes à des fins thérapeutiques est une pratique multiséculaire (Gbénou et al., 2011). Les plantes médicinales, une des principales composantes des Produits Forestiers Non Ligneux (PFNL), constituent des ressources précieuses pour la grande majorité des populations rurales des pays en développement (Guedje et al., 2010). L'espèce Bixa orellana est utilisée dans divers traitements traditionnels par les populations locales. Ainsi, les racines et les feuilles de Bixa orellana sont utilisées pour traiter l'épilepsie, la diarrhée, la dysenterie, la fièvre, l'ictère, les maladies parasitaires, la toux et les infections urogénitales, certaines maladies gastro-intestinales et pulmonaires (Giorgi et al., 2013; de Araújo Vilar et al., 2014) dans plusieurs pays notamment au Brésil, au Pérou, en Philippine, au Nigéria et en Côte d'Ivoire (Omonhinmin et al., 2013; de Araújo Vilar et al., 2014; Koffi, 2015). Le traitement des nombreuses maladies par les organes de l'espèce se baserait sur leurs différentes propriétés biologiques (Tableau 1).

Par ailleurs, différents extraits des feuilles, graines et racines de Bixa orellana ont révélé la présence de nombreux composés phytochimiques tels que les alcaloïdes, les tanins, les flavonoïdes, les saponosides, les composés réducteurs, les dérivés quinoniques, les coumarines, les terpènoides, les triterpènoïdes et stéroïdes, les dérivés cyanogéniques, les anthocyanes et les hétérosides cardiotoniques (Fleischer et al., 2003; Quanico et al., 2008; Sangvikar et al., 2015; Dike et al., 2016; Prathima et al., 2016). Ces métabolites secondaires sont connus comme possédant diverses propriétés pharmacologiques et pourraient donc être responsables des activités biologiques mises en évidences (Déléké Koko et al., 2011).

\section{Importance alimentaire}

Bixa orellana est une plante à usages multiples. Cependant, de tous les organes de l'arbre, les graines rouges appelées roucou ou 
rocou en français, annatto en anglais et achiote en espagnol, sont les plus utilisées (Venugopalan et al., 2011; Valentin et al., 2013). Les graines de Bixa orellana sont utilisées dans diverses préparations alimentaires par les populations des pays en développement (de Araújo Vilar et al., 2014). Par ailleurs, les graines de Bixa orellana constituent une importante source de nutriments qui, incorporées à divers aliments pourraient contribuer à leur enrichissement en substances nutritives et réduire les risques de maladies liées à la consommation d'aliments carencés surtout dans plusieurs pays en développement (Senthil et al., 2007; del Prado et al., 2009).

Bixa orellana constitue donc une source pourvoyeuse de devise pour une nation à travers sa production, sa transformation et son exportation mais aussi contribue à l'amélioration de l'alimentation à travers sa consommation compte tenu de sa composition biochimique.

\section{COMPOSITION BIOCHIMIQUE DES GRAINES DE BIXA ORELLANA}

Les graines de Bixa orellana constituent une importante source de certains nutriments au regard de sa composition (Tableau 2). En effet, il a été rapporté que le taux de protéines dans les graines de roucou varie de 10,6 (Glew et al., 1997) à 17,6\% (Kapoor et al., 1975) avec une moyenne de $13,7 \%$. Une variation a été aussi observée au niveau de la teneur en lipides totaux des graines qui est comprise entre 2,23\% (Valério et al., 2015) et 7,20\% (Dike et al., 2016). La teneur en hydrates de carbone et en fibre brute varie respectivement de 16,26\% (Dike et al., 2016) à 78,11\% (Moraes, 2007) et de $13,84 \%$ (Silva, 2010) à 53,31\% (Dike et al., 2016) avec une moyenne respective de $45,52 \%$ et de $31,39 \%$. Globalement, la valeur énergétique du péricarpe des graines de Bixa orellana, considérée jusqu'à un passé récent comme une perte, a été estimée à $16 \mathrm{MJ} / \mathrm{Kg}$ ce qui attribue des applications plus larges au Bixa orellana qui vont au-delà de leurs valeurs traditionnelles comme colorant alimentaire (Parimalan et al., 2007). Aussi, Valério et al., (2015) ont constaté que les graines de Bixa orellana possèdent une valeur énergétique totale estimée à $234,46 \mathrm{kcal} / 100 \mathrm{~g}$. La faible teneur en cendre totale des graines de roucou a été trouvée par Kapoor et al., (1975) alors que la valeur élevée de cendre totale a été rapportée par Pereira et al., (2009) avec une teneur moyenne de 5,20\% (Tableau 2). Les minéraux présents dans les graines de Bixa orellana ont été révélés par les travaux effectués par Ferreira et Falesi (1989), Anselmo et al., (2008) et Valério et al., (2015). Ainsi, on note la présence de sodium, de calcium, de potassium, de magnésium, de manganèse de fer, du cuivre et du zinc à diverses proportions (Tableau 2).

\section{PROPRIETES TECHNOLOGIQUES ET APPLICATIONS DU COLORANT DE BIXA ORELLANA}

Les colorants naturels sont connus pour leur usage dans la coloration des aliments, des cuirs et des fibres naturelles (Madiélé et al., 2015). Un colorant alimentaire doit avoir des caractéristiques telles qu'un pouvoir colorant élevé, une grande stabilité dans des conditions spécifiques d'utilisation ( $\mathrm{pH}$, température), une gamme de couleurs précise et stable d'un batch de production à l'autre. Le colorant extrait des graines de Bixa orellana est constitué de deux fractions dont une fraction liposoluble (la bixine) et une fraction hydrosoluble (la norbixine). Les graines de Bixa orellana constituent la seule source naturelle de la bixine, un caroténoïde très largement utilisé dans l'industrie alimentaire comme colorant. Les avantages des caroténoïdes en tant que colorants sont leur stabilité aux différents $\mathrm{pH}(4,1-7,0)$ de nombreux aliments et le fait que l'addition des différents additifs n'affecte pas leur structure (Kusdiyantini, 1998). La forte puissance de coloration des caroténoïdes justifie leur large utilisation (Soumya et al., 2010). Les caroténoïdes ont été intensivement étudié à cause de leurs fonctions biologiques connues, telles que leurs propriétés provitaminique (vitamine A), antioxydante et antiradicalaire, des effets empêchant le cancer, l'effet protecteur contre les maladies cardiovasculaires et réduisant le risque de cataracte et la dégénération moléculaire relative à l'âge 
(Van den Berg et al., 2000). L'application des colorants naturels en alimentation montera dans un proche avenir avec une plus grande conscience du consommateur et des restrictions législatives croissantes contre l'utilisation des colorants de synthèse (Prabhakara Rao et al., 2004). La stabilité de ces différentes fractions de colorants naturels extraites des graines de Bixa orellana a été étudiée pendant la cuisson dans la farine du riz par Maga et Kim (1990). La pâte a été retirée aux températures de $125-155{ }^{\circ} \mathrm{C}$ avec les concentrations de $0,1 \mathrm{~g} / 100 \mathrm{~g}$ de colorant hydrosoluble et de $0,3 \mathrm{~g} / 100 \mathrm{~g}$ du colorant liposoluble. L'analyse par la méthode de réflectivité ou par chromatographie sur couche mince et par spectroscopie a prouvé que les colorants ont subi une certaine perte pendant la cuisson à une température élevée, et le colorant hydrosoluble s'est avéré plus stable. Une étude similaire réalisée par Berset et Marty (1986) incorporant 15-45 ppm de rocou dans l'amidon du maïs et la cuisson à 175-185 ${ }^{\circ} \mathrm{C}$ a prouvé la stabilité thermique du colorant. La stabilité de la couleur des solutions hydrosolubles commerciales de rocouyer a été étudiée en les exposant aux températures de chauffage de $90,100,120$ et $140{ }^{\circ} \mathrm{C}$ pour des durées allant jusqu'à 450 minutes ( 7 Heures 30 minutes) (Ferreira et al., 1999). Dans cette étude, les variations des caractéristiques de couleur et des concentrations de norbixine ont été déterminées. Il s'est avéré que pendant le chauffage, la couleur jaune a augmenté et celle rouge a diminué; et la cinétique a montré que la dégradation des caractéristiques de la bixine a suivi une réaction de premier ordre tandis que la dégradation de la norbixine était une réaction de second ordre.

Les études effectuées par Prabhakara Rao et al., (2004) ont prouvé que le colorant liposoluble des graines de Bixa orellana (bixine) pourrait efficacement être utilisé dans la plupart des produits de boulangerie et des produits de grande consommation tels que les frites de courte durée pour donner à l'aliment, une couleur attrayante à la vue. Dans toutes leurs expériences, les pertes ont augmenté avec l'augmentation des concentrations appliquées. La conservation du colorant était tout à fait élevée aux températures inférieures à $180{ }^{\circ} \mathrm{C}$. En cas d'une expérience de chauffage profond en huile, les pertes sont élevées dans le produit ce qui est dû à la lixiviation $\mathrm{du}$ colorant par l'huile. Ce problème peut être obvié en utilisant le colorant hydrosoluble (norbixine) pour empêcher la lixiviation du colorant par l'huile. L'application de la norbixine donnerait une couleur uniforme au riz frit. Ces mêmes auteurs ont ajouté que pendant la préparation de différents produits, la perte en bixine était semblable à un traitement au four ainsi qu'à une cuisson à la pression mais elle était négligeable une fois exposée aux microondes. Il ressort donc des travaux de Prabhakara Rao et al., (2004) que les pertes de bixine dans le colorant étaient plus élevées lorsque le colorant est directement exposé aux conditions de traitement que lorsqu'il est incorporé aux produits traités. L'utilisation des colorants des graines de Bixa orellana nécessite leur extraction. A cet effet, différentes méthodes essentiellement chimiques ont été utilisées pour extraire ces colorants mais avec des rendements variables d'une méthode à une autre. Le Tableau 3 cidessous nous renseigne sur certaines de ces méthodes.

\section{PRINCIPALES VOIES DE VALORISATION DE BIXA ORELLANA}

Bixa orellana est une espèce tinctoriale dont les voies de valorisation sont multiples. Dans la région amazonienne, les graines sont utilisées comme épice ou condiment. En outre elles sont utilisées comme colorant naturel dans une variété de produits alimentaires. $\mathrm{La}$ plupart du temps, les graines sont vendues soit réduites en poudre soit en pâte d'extrait produite avec les huiles végétales ou les bases chimiques (Lim, 2011). De nombreux groupes socio-culturels utilisent le colorant naturel des graines pour colorer les céramiques et d'autres ustensiles pour des usages domestiques. Ils l'utilisent aussi pour se colorer la peau pendant des rituels religieux et pour se protéger contre la lumière solaire et contre des moustiques (Barbosa-Filho et al., 1988). Les graines sont données aux taureaux pour les rendre agressifs pour des combats de taureaux et sont aussi utilisées comme laxatif, hypotensif, fluidifiant, 
et antimicrobien. Aussi, les graines sont considérées en médecine traditionnelle comme possédant une activité anti-inflammatoire pour des contusions et des blessures et l'infusion des feuilles est utilisée contre la bronchite, les maux de gorge et l'inflammation des yeux (de Araújo Vilar et al., 2014). En dépit de la différence existant entre les cultures et les traditions, les utilisations traditionnelles des graines de Bixa orellana sont les mêmes, comme colorant, condiment, antipyrétique, aphrodisiaque, anti-diarrhéique, antidiabétique et révulsif des insectes (de Araújo Vilar, 2014 ; Sangvikar et al., 2015).

Les graines de Bixa orellana fournissent l'un des colorants le plus fréquemment utilisé dans le monde entier, non seulement dans des produits alimentaires mais également dans le textile, la peinture et les industries cosmétiques. Son utilisation a été stimulée suite à l'interdiction de l'utilisation des colorants de synthèse en alimentation et produits cosmétiques, et il est l'un des colorants acceptés par l'OMS, car, en plus d'être nontoxique, il semble ne pas modifier la valeur nutritive de l'aliment (Bastos et al., 1999). Par ailleurs, un autre fait intéressant est que $70 \%$, de tous les agents naturels de coloration, consommés dans le monde entier dérivent du Bixa orellana (Thomas et al., 2005). Les graines de Bixa orellana sont donc utilisées dans plusieurs domaines. Leur utilisation dans l'agroalimentaire est d'autant plus intéressante du moment où les colorants de synthèses sont soupçonnés comme causes de nombreuses maladies telles que le cancer de la thyroïde, l'influence sur les fonctions nerveuses, les tumeurs rénales, la maladie d'Alzheimer, la perturbation de la formule sanguine (Arzour et Belbacha, 2015). La valorisation donc de ces graines dans les domaines agroalimentaires, cosmétiques et pharmaceutiques est envisageable.

\section{PERSPECTIVES DE VALORISATION DE BIXA ORELLANA AU BENIN}

Aujourd'hui, au regard des réalités socio-économiques des divers pays en développement (revenus insuffisants, rareté d'emploi, insécurité alimentaire chronique, etc.), la population a développé des activités lucratives diversifiées pour se maintenir, parmi lesquelles l'exploitation des Produits Forestiers Non Ligneux (PFNL) (Biloso et Lejoly, 2006). L'exploitation judicieuse des PFNL est de plus en plus considérée comme une stratégie de conservation des ressources tropicales, en même temps qu'elle permet une amélioration du bien-être des populations locales forestières par la lutte contre la pauvreté (Guedje, 2004). La graine de Bixa orellana, étant également un produit forestier non ligneux, il sera d'une utilité économique de la valoriser au plan technologique à travers l'extraction des colorants, la caractérisation de ceux-ci et des essais quant à leurs applications pratiques. Les colorants artificiels ou de synthèse étant des causes de sérieux problèmes de santé (Downham et Collins, 2000), la demande des consommateurs et de l'industrie alimentaire pour l'utilisation de pigments naturels extraits par des procédés biologiques est en grande expansion (Ligia Alves, 2008). Les procédés existants pour l'extraction du colorant des graines de Bixa orellana sont essentiellement chimiques et industriels (da Costa et Chaves, 2005), nécessitant de lourds équipements et ne sont pas utilisables dans l'artisanat local. La production du colorant des graines de Bixa orellana par des méthodes simples et efficaces sans additif chimique et son utilisation dans diverses formulations contribueront significativement à sa valorisation. Pour une utilisation durable et efficace de Bixa orellana dans le monde en général et au Bénin en particulier, il serait donc nécessaire de mettre au point une technologie optimale d'extraction des colorants des graines de Bixa orellana tout en préservant leurs propriétés antioxydantes et antimicrobiennes pour diverses utilisations.

\section{SYNTHESE ANALYTIQUE}

Il ressort des informations collectées que le roucouyer (Bixa orellana), principale source de bixine, un biocolorant largement utilisé en industries alimentaires, est cultivée dans de nombreuses régions tropicales (Fagbohoun, 2014; de Araújo Vilar et al., 2014, U1-Islam et al., 2016, Periyasamy et al., 2016). Sa production mondiale n'est 
cependant pas encore suffisante pour remplacer efficacement les colorants de synthèse de même couleur utilisés dans le domaine alimentaire. La production de Bixa orellana peut donc constituer une source de devise pour les nations en développement et surtout permettre aux pays développés industrialisés d'utiliser du colorant biologique répondant aux critères normatifs, aux préférences des consommateurs et aux différentes conditions des produits qu'ils fabriquent. Les graines de Bixa orellana constituent une importante source de certains nutriments à travers leur composition (Valério et al., 2015; Dike et al., 2016). Ainsi, l'incorporation d'extraits ou de poudre de ces graines à certains aliments pourrait accroître leur valeur nutritive et donc limiter le développement de certaines maladies nutritionnelles liées aux carences en substances nutritives dans les pays en développement. Le colorant des graines de Bixa orellana peut être extrait par diverses méthodes qui ont été développées et expérimentées. Toutefois, il est à remarquer que ces méthodes sont pour la plupart basées sur l'utilisation des réactifs chimiques et ou d'équipements industriels assez pointus qui ne sont pas accessibles aux petits exploitants ou à des populations rurales (da Costa et Chaves, 2005). Il serait donc plus profitable aux populations rurales que des méthodes simples n'utilisant pas de solvants chimiques et d'équipements lourds soient mises en œuvre afin de permettre également à cette couche de bénéficier des avantages qu'offrent les graines de Bixa orellana en tant que source de biocolorant.

Les principaux colorants extraits des graines de Bixa orellana sont la bixine liposoluble et la norbixine, hydrosoluble (Yolmeh et al., 2014; Zarza-García et al., 2017). Ces deux composés sont mieux conservés dans les différents produits lorsque les températures maximales de cuissons des produits sont inférieures à $180^{\circ} \mathrm{C}$ (Prabhakara Rao et al., 2004). Il ressort donc que les températures élevées favorisent les pertes du colorant soit par dégradation du colorant en d'autres produits soit par dénaturation. Les pertes de bixine dans le colorant sont donc plus élevées lorsque le colorant est directement exposé aux températures élevées (supérieures à $180{ }^{\circ} \mathrm{C}$ ) que lorsqu'il est incorporé aux produits traités (Prabhakara Rao et al., 2004). Le roucouyer (Bixa orellana) est une espèce tinctoriale dont les voies de valorisation sont multiples. Différentes parties de l'espèce sont utilisées surtout pour les traitements médicinaux et les graines sont utilisées dans diverses formulations alimentaires (Yolmeh et al., 2014; Taham et al., 2015). Sa valorisation passe donc par la vulgarisation des travaux de recherches qui serviront d'une part de support à la communauté scientifique pour l'approfondissement de thématiques de recherche beaucoup plus pointues, mais aussi aux petits et grands industriels qui prendront connaissance des valeurs dont regorge Bixa orellana à travers son biocolorant. L'utilisation des graines dans l'agroalimentaire est d'autant plus intéressante du moment où les colorants de synthèse sont soupçonnés comme causes de nombreuses maladies. Tout ceci en synergie avec les exploitants qui sont les premiers acteurs de la chaîne et qui devront bénéficier de leurs productions pour une pérennisation de la culture. La production du colorant des graines de Bixa orellana par des méthodes simples et efficaces sans additifs chimiques et son utilisation dans diverses formulations contribueront significativement à sa valorisation. Pour une utilisation durable et efficace de Bixa orellana au Bénin, il serait donc nécessaire de promouvoir sa culture et de mettre au point une technologie optimale d'extraction des colorants des graines de Bixa orellana tout en préservant leurs propriétés antioxydantes et antimicrobiennes pour diverses utilisations. 

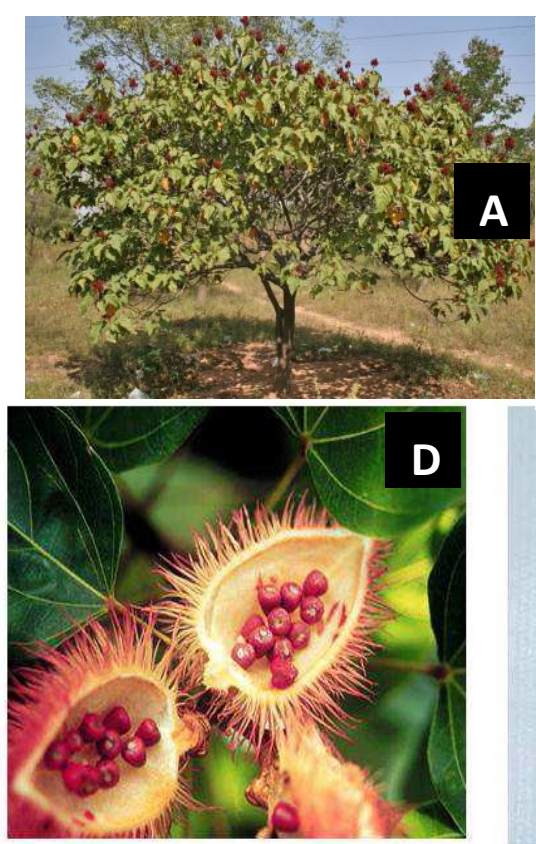
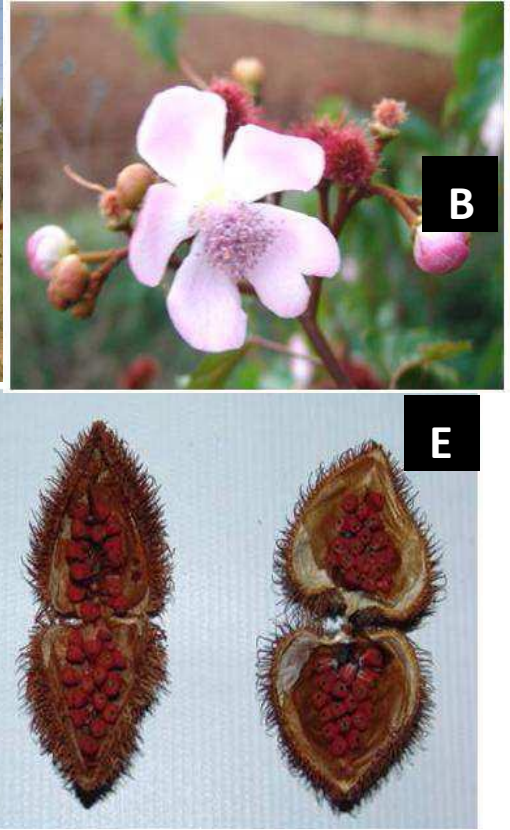
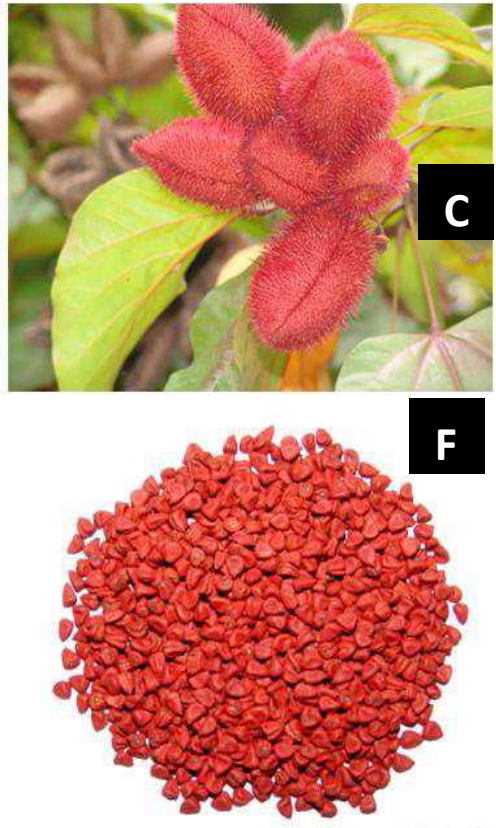

Figure 1: Bixa orellana: (A) Arbre; (B): Fleures; (C): Grappe de fruits; (D): Fruit immature; (E): Fruits déhiscents avec des graines à l'intérieur; (F): Graines (Karen, 2014; Giridhar et al., 2014).

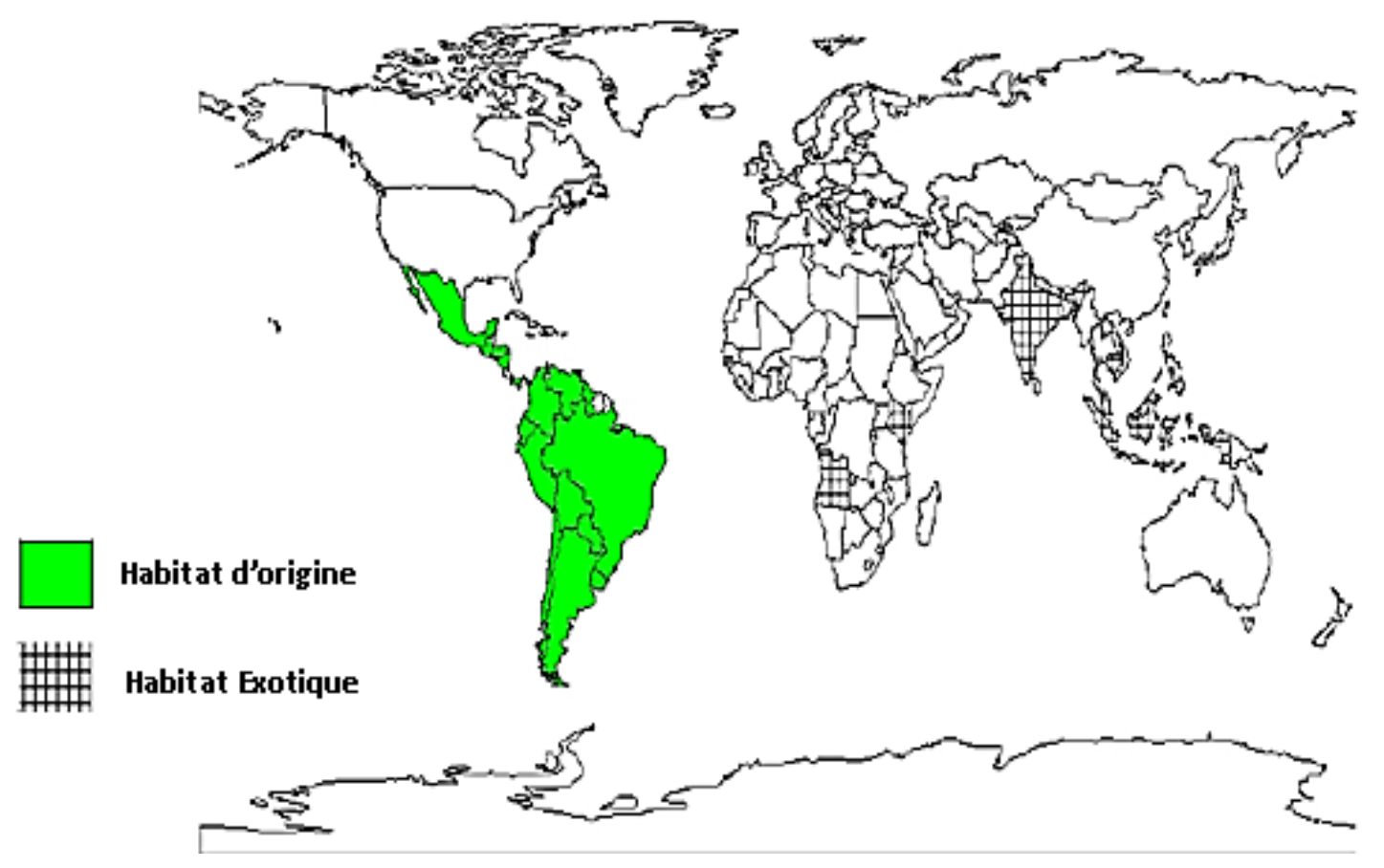

Figure 2: Répartition mondiale du Bixa orellana (Orwa et al., 2009). 
Zone de faible présence

Zone de forte présence

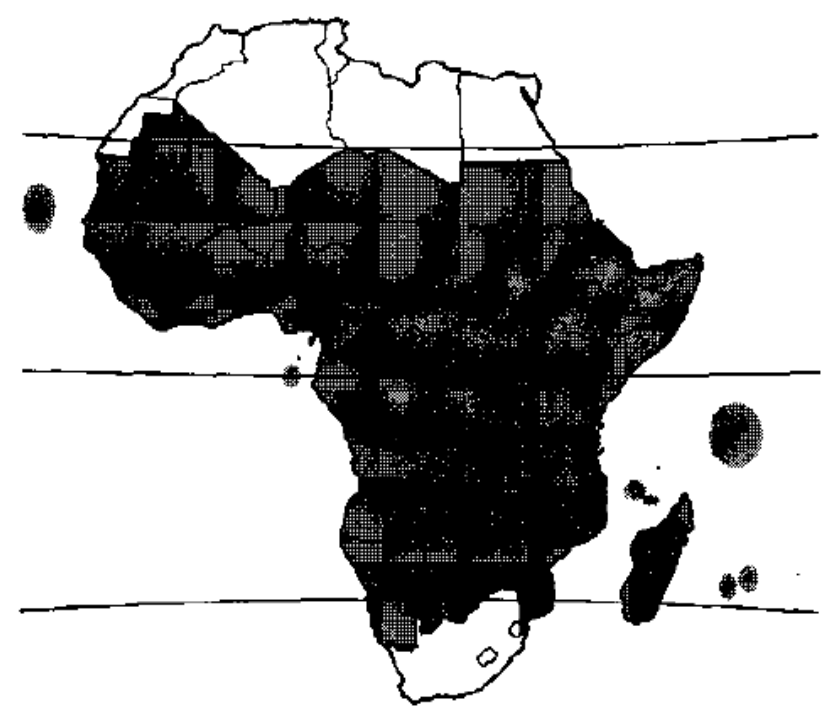

Figure 3: Répartition de la culture du Bixa orellana en Afrique (Jansen, 2005).

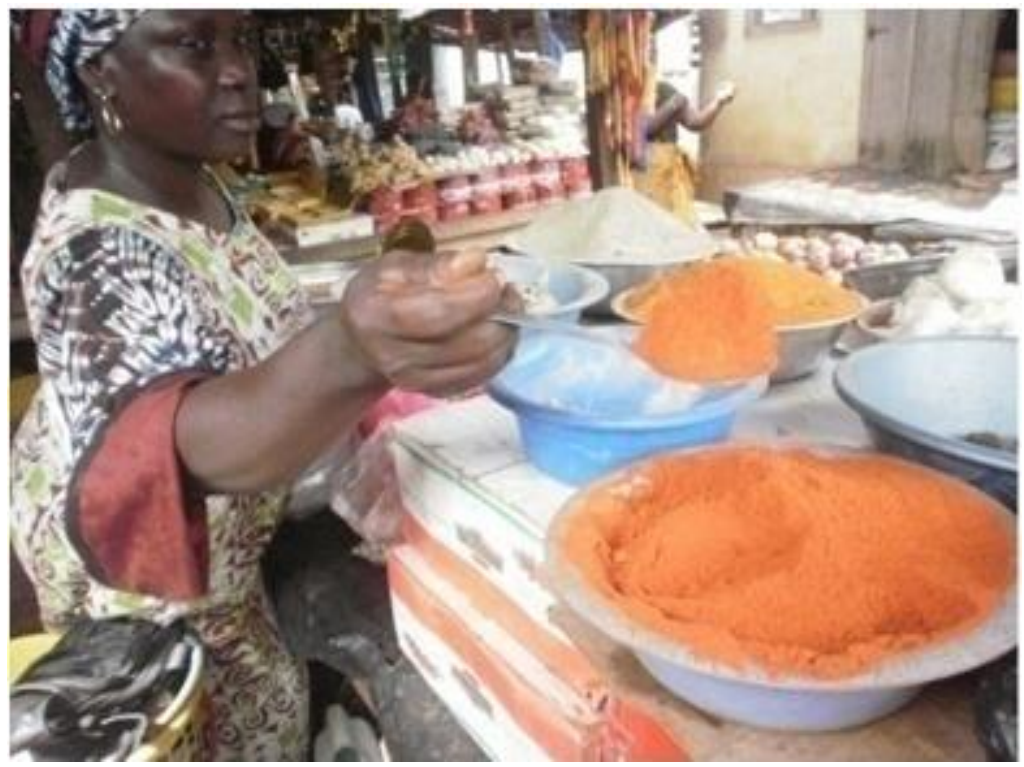

Figure 4 : Vendeuse de poudre de roucou ou tomate mougou au marché de Tanda (Koffi, 2015). 
Tableau 1: Activités biologiques de quelques organes de l'espèce Bixa orellana.

\begin{tabular}{|c|c|c|c|c|c|}
\hline Pays & $\begin{array}{l}\text { Organes } \\
\text { utilisés }\end{array}$ & $\begin{array}{l}\text { Type de } \\
\text { solvants }\end{array}$ & $\begin{array}{l}\text { Activités biologiques } \\
\text { mises en évidence }\end{array}$ & Degré d'activité & Références \\
\hline Brésil & Graines & Ethanol & Antioxydante & La concentration de $0,1 \mathrm{~g} / \mathrm{L}$ d'extrait a montré une activité antioxydante & Giorgi et al., 2013 \\
\hline Cuba & Graines & Ethanol & Cytotoxicité & $\begin{array}{l}\text { L'extrait éthanolique des graines est cytotoxique pour les cellules tumorales avec une concentration allant de } 3,9-250 \\
\mathrm{mg} / \mathrm{mL}\end{array}$ & Garcia et al., 2011 \\
\hline Brésil & Graines & Eau & Hyperlipidémie & Actif à une dose de $400-800 \mathrm{mg} / \mathrm{kg}$ lorsqu'il est administré à une souris & Ferreira et al., 2013 \\
\hline Brésil & Graines & - & \multirow{3}{*}{ Antileishmaniale } & Actif sur Leishmania amazonensis à des concentrations de 10/50/100/500/1000 $\mu \mathrm{g} / \mathrm{mL}$ & Lopes et al., 2012 \\
\hline Brésil & Feuilles/Racine & Ethanol & & \multirow{2}{*}{$\begin{array}{l}\text { Actif sur Leishmania amazonensis à des concentrations de } 0,12-2,5 \mathrm{mg} / \mathrm{mL} \\
\mathrm{L} \text { 'extrait méthanolique des feuilles présente une activité Antifongique sur Trichophyton mentagrophytes à une dose de } 10 \\
\mathrm{mg} / \text { disque }\end{array}$} & Almeida et al., 2012 \\
\hline Equateur & Feuilles & Méthanol & & & Freixa et al., 1998 \\
\hline Nigéria & Feuilles & Ethanol & & $\begin{array}{l}\text { Les extraits de feuilles sont actifs avec une concentration de } 4,0 \mathrm{mg} / \mathrm{mL} \text { contre Bacillus subtilis et sur Staphylococcus } \\
\text { aureus mais avec une concentrationde } 16,0 \mathrm{mg} / \mathrm{mL} \text { pour Streptococcus faecalis }\end{array}$ & Irobiet al., 1996 \\
\hline Ghana & Feuilles/Graines & Ethanol & Antimicrobienne & $\begin{array}{l}\text { Activité antibactérienne de large spectre avec un diamètre d'inhibition de } 21,50 ; 20,00 ; 19,50 ; 17,00 ; 19,00 ; 22,50 \text { et de } \\
22,00 \mathrm{~mm} \text { pour les extraits de feuilles et des diamètres d'inhibition de } 20,00 ; 17,00 ; 19,00 ; 14,50 ; 19,00 ; 18,00 \text { et } 20,00 \\
\text { mm pour les extraits de graines respectivement contre Bacillus subtilis, Staphylococcus aureus, Streptococcus pyogenes, } \\
\text { Salmonella typhi, Pseudomonas aeruginosa, Escherichia coli et Candida albicans. }\end{array}$ & Fleischer et al., 2003 \\
\hline Thailand & Feuilles & & & Actif contre Staphylococcus aureus avec une concentration minimale d'inhibition de $62,5 \mu \mathrm{g} / \mathrm{ml}$ & Metta et al., 2009 \\
\hline Inde & Feuilles & & & $\begin{array}{l}\text { L'extrait éthanolique des feuilles a montré une activité inhibitrice avec les concentrations minimales inhibitrices de 0,62; } \\
2,5 ; 1,25 \text { et } 0,62 \mathrm{mg} / \mathrm{ml} \text { respectivement sur Staphylococcus aureus; Pseudomonas Spp, Bcillus Subtilis et Escherichia Coli. }\end{array}$ & Rajendra, 2014 \\
\hline \multirow{2}{*}{ Bangladesh } & Graines & Méthanol & $\begin{array}{l}\text { Hypocholestérolé- } \\
\text { miante }\end{array}$ & $\begin{array}{l}\text { L'extrait méthanolique des graines à raison de } 500 \mathrm{mg} / \mathrm{kg} \text { de poids corporel présente une activité hypocholestérolémiante } \\
\text { de } 52.90 \% \text { et une dose de } 250 \mathrm{mg} / \mathrm{kg} \text { induit une réduction de } 38.32 \%\end{array}$ & \multirow{2}{*}{ Rajib et al., 2009} \\
\hline & Graines & & Hypoglycémiante & $\begin{array}{l}\text { L'extrait méthanolique des graines à raison de } 500 \mathrm{mg} / \mathrm{kg} \text { de poids corporel induit une réduction de } 2,20 \% \text { du taux de } \\
\text { glucose et la dose de } 250 \mathrm{mg} / \mathrm{kg} \text { induit une réduction de } 1,65 \% \text { du taux de glucose }\end{array}$ & \\
\hline
\end{tabular}


Tableau 2: Composition biochimique des graines de Bixa orellana.

\begin{tabular}{|c|c|c|c|c|}
\hline \multirow{2}{*}{ Constituants } & \multicolumn{3}{|c|}{ Valeurs } & \multirow{2}{*}{ Références } \\
\hline & Min & Moy & Max & \\
\hline \multicolumn{5}{|l|}{ Macronutriments } \\
\hline $\begin{array}{l}\text { Protéines brutes } \\
(\mathrm{g} / 100 \mathrm{~g} \mathrm{MS})\end{array}$ & $10,6 \%$ & $13,69 \%$ & $17,6 \%$ & $\begin{array}{l}\text { Kapoor et al., (1975); } \\
\text { Senthil et al., (2007); } \\
\text { Wurtset al., (1983); } \\
\text { Bressaniet al., (1983); } \\
\text { Glewetal., (1997); } \\
\text { da Silva et al., (2009); } \\
\text { Utiyama et al., (2002); } \\
\text { Anselmo et al., (2008); } \\
\text { Valéria et al., (2015); } \\
\text { Carvalho et al., (1991); } \\
\text { Pedrosa et al.,(1999); } \\
\text { Dike et al., (2016); }\end{array}$ \\
\hline $\begin{array}{l}\text { Lipides totaux } \\
\text { (g/100g MS) }\end{array}$ & $2,23 \%$ & $5 \%$ & $7,20 \pm 0,07$ & $\begin{array}{l}\text { Alonso et al., (2009); } \\
\text { Valério et al., (2015); } \\
\text { Dike et al., (2016) }\end{array}$ \\
\hline $\begin{array}{l}\text { Hydrates de } \\
\text { carbone }(\mathrm{g} / 100 \mathrm{~g} \\
\mathrm{MS}) \\
\end{array}$ & $16,26 \%$ & $45,52 \%$ & $78,11 \%$ & $\begin{array}{l}\text { Valério et al., (2015); } \\
\text { Moraes (2007); } \\
\text { Dike et al., (2016) }\end{array}$ \\
\hline $\begin{array}{l}\text { Fibres brutes } \\
(\mathrm{g} / 100 \mathrm{~g} \mathrm{MS})\end{array}$ & $13,84 \%$ & $31,39 \%$ & $53,31 \pm 0,07 \%$ & $\begin{array}{l}\text { Valério et al., (2015); } \\
\text { da Silva (2009); } \\
\text { Senthil et al., (2007); } \\
\text { Utiyama et al., (2002); } \\
\text { Bressani et al., (1983); } \\
\text { Anselmo et al., (2008); } \\
\text { Dike et al., (2016); }\end{array}$ \\
\hline $\begin{array}{l}\text { Energie (kcal/100g } \\
\text { MS) }\end{array}$ & $16 \mathrm{MJ} / \mathrm{Kg}$ & - & $234,46 \mathrm{kcal} / 100 \mathrm{~g}$ & $\begin{array}{l}\text { Parimalan et al., (2007); } \\
\text { Valério et al., (2015) }\end{array}$ \\
\hline $\begin{array}{l}\text { Cendre totale } \\
(\mathrm{g} / 100 \mathrm{~g} \mathrm{MS})\end{array}$ & $4,7 \%$ & $5,20 \%$ & $6,32 \%$ & $\begin{array}{l}\text { Senthil et al., (2007). } \\
\text { Kapoor et al., (1975). } \\
\text { Alonso, (1998); } \\
\text { da Silva et al., (2009); } \\
\text { Moraes (2007); } \\
\text { Utiyama et al., (2002); } \\
\text { Pereira et al., (2009); } \\
\text { Valério et al., (2015); } \\
\text { Dike et al., (2016); }\end{array}$ \\
\hline \multicolumn{5}{|c|}{ Minéraux individuels (mg/100g MS) } \\
\hline $\mathrm{Na}$ & $35.61 \mathrm{mg} \cdot \mathrm{g}^{-1}$ & - & & Valério et al., (2015) \\
\hline $\mathrm{Ca}$ & $0,07 \mathrm{mg} \cdot \mathrm{g}^{-1}$ & - & $1,82 \mathrm{mg} \cdot \mathrm{g}^{-1}$ & $\begin{array}{l}\text { Valério et al., (2015); } \\
\text { Anselmo et al., (2008); } \\
\text { Ferreira et Falesi (1989). }\end{array}$ \\
\hline $\mathrm{K}$ & $19.2 ; \mathrm{mg} . \mathrm{g}^{-1}$ & - & $70.77 \mathrm{mg} . \mathrm{g}^{-1}$ & $\begin{array}{l}\text { Valério et al., (2015); } \\
\text { Ferreira et Falesi (1989). }\end{array}$ \\
\hline $\mathrm{Mg}$ & $0.03 \mathrm{mg} \cdot \mathrm{g}^{-1}$ & - & $2,22 \mathrm{mg} \cdot \mathrm{g}^{-1}$ & Valério et al., (2015); \\
\hline
\end{tabular}




\begin{tabular}{|c|c|c|c|c|}
\hline & & & & Ferreira et Falesi (1989). \\
\hline $\mathrm{Mn}$ & $0,023 \mathrm{mg} \cdot \mathrm{g}^{-1}$ & - & $0.25 \mathrm{mg} \cdot \mathrm{g}^{-1}$ & $\begin{array}{l}\text { Valério et al., (2015); } \\
\text { Ferreira et Falesi (1989) }\end{array}$ \\
\hline $\mathrm{Fe}$ & $0,008 \mathrm{mg} \cdot \mathrm{g}^{-1}$ & - & $0.03 \mathrm{mg} \cdot \mathrm{g}^{-1}$ & $\begin{array}{l}\text { Valério et al., (2015); } \\
\text { Anselmo et al., (2008). }\end{array}$ \\
\hline $\mathrm{Cu}$ & $0,0046 \mathrm{mg} \cdot \mathrm{g}^{-1}$ & - & $0,03 \mathrm{mg} \cdot \mathrm{g}^{-1}$ & $\begin{array}{l}\text { Valério et al., (2015); } \\
\text { Ferreira et Falesi (1989) }\end{array}$ \\
\hline $\mathrm{Zn}$ & $0,0354 \mathrm{mg} \cdot \mathrm{g}^{-1}$ & - & & Ferreira et Falesi (1989) \\
\hline
\end{tabular}

Tableau 3: Rendements d'extraction en fonction des méthodes d'extraction de colorants des graines de Bixa orellana (Giridhar et al., 2014).

\begin{tabular}{|c|c|c|}
\hline $\mathbf{N}^{\circ}$ & Type de méthode d'extraction & Rendement et pureté \\
\hline 1 & $\begin{array}{l}\text { Extraction du colorant des graines au Chloroforme } \\
(1: 5)\end{array}$ & $\begin{array}{l}1,6 \% \text { de cristaux de bixine pure (sur la base du } \\
\text { poids de graine), jusqu'à 59\% des colorants totaux }\end{array}$ \\
\hline 2 & Ebullition avec de l'acétate éthylique & $1,1 \%$ de cristaux de bixine \\
\hline 3 & Méthode critique superbe de $\mathrm{CO}_{2}$ & $\sim 1 \%$ de colorant \\
\hline 4 & $\begin{array}{l}\text { Méthode critique superbe de } \mathrm{CO}_{2} \text { avec une } \\
\text { combinaison des modes statiques et dynamiques } \\
\text { d'extrait avec du } \mathrm{CO}_{2}\end{array}$ & 2,7 mg de bixine par g. d.w. \\
\hline 5 & $\begin{array}{l}\text { Extraction et purification de bixine par agitation } \\
\text { mécanique et au solvant }\end{array}$ & $18,6 \%$ de Bixine pure \\
\hline & Séchage par jet du lit d'extrait aqueux de rocouyer & $\begin{array}{l}\text { Rendement élevé de poudre ultrafine du colorant } \\
\sim 15 \%\end{array}$ \\
\hline 6 & Méthode d'huile de roulette & $13,25 \%$ colorant total \\
\hline 7 & Méthode colloïdale d'aphrons de gaz & $\begin{array}{l}3,26 \%(\mathrm{w} / \mathrm{w}) \text { de norbixine dans les graines } \\
\text { Rendement d'extrait } 81 \% \\
94 \% \text { d'extrait de norbixine récupéré }\end{array}$ \\
\hline 8 & Extrait supercritique de $\mathrm{CO}_{2}$ avec de l'éthanol & $\begin{array}{l}45 \% \text { de récupération de bixine } \\
13,7 \mathrm{~g} / \mathrm{dm} 3 \text { de colorant extractible }\end{array}$ \\
\hline 9 & Extraction de bixine en utilisant le $\mathrm{CO}_{2}$ supercritique & La solubilité de $93 \%$ de bixine pure est réalisée \\
\hline 10 & Méthode de jet de lit & 15-24\% de bixine récupéré \\
\hline 11 & $\begin{array}{l}\text { Méthode de solvant utilisant du Chloroforme et le } \\
\text { dichlorométhane }\end{array}$ & 5 - 7\% de colorant total de B. Orellana (w/w) \\
\hline 12 & $\begin{array}{l}\text { Extraction avec du chloroforme suivi d'un traitement } \\
\text { alcalin }\end{array}$ & Rendement de norbixine de $10 \%$ \\
\hline 13 & $\begin{array}{l}\text { Méthode améliorée pour l'extrait de bixine et la qualité } \\
\text { de rendement }\end{array}$ & Bixine fortement purifiée \\
\hline 14 & $\begin{array}{l}\text { Traitement en aval des graines du rocouyer en utilisant } \\
\text { la méthode du solvant }\end{array}$ & $\begin{array}{l}3 \% \text { de cristaux purs de la bixine (w/w) du colorant } \\
\text { cristallin qui contient } \sim 1 \% \text { de bixine (à base du } \\
\text { poids de graine) }\end{array}$ \\
\hline 15 & Méthode de Séchage au vaporisateur & Rendement élevé de norbixine \\
\hline 16 & $\begin{array}{l}\text { Extraction Assistée à la micro-onde du colorant } \\
\text { normal des graines de Bixa orellana à l'aide des } \\
\text { modèles MRS et ANN }\end{array}$ & $\begin{array}{l}\text { Possibilité d'extraction efficace du colorant de } \\
\text { rocouyer }\end{array}$ \\
\hline 17 & $\begin{array}{l}\text { Séparation de norbixine du colorant cru obtenu des } \\
\text { graines de Bixa orellana }\end{array}$ & 99\% de norbixine récupérée \\
\hline 18 & Méthode d'extraction aqueuse à deux phases & Purification de la norbixine \\
\hline
\end{tabular}




\section{Conclusion}

Les différentes informations discutées dans cette revue représentent une base de données sur les propriétés de Bixa orellana et sur ses utilisations dans divers domaines. Ces études renseignent sur la production de l'espèce au plan international et régional; et sur son importance socio-économique et nutritionnelle. Elles révèlent également les propriétés pharmacologiques et technologiques du colorant extrait des graines de Bixa orellana et met en exergue les défis pour la valorisation de l'espèce. Différents organes de l'espèce sont utilisés à des fins économiques, médicinales, cosmétiques et alimentaires. $\mathrm{Au}$ regard de toutes ces informations, il faudra identifier des variétés ou écotypes de Bixa orellana performants du point de vue de la qualité et de la quantité des graines pour une production massive en vue de leur valorisation technologique et agroalimentaire. Ainsi, comme défis, il faudra procéder à une caractérisation au plan ethnobotanique, génétique, morphologique, agronomique de même que le potentiel de régénération de Bixa orellana d'écologie béninoise. Déterminer les caractéristiques physicochimiques et génétiques des graines de Bixa orellana, mettre au point un procédé biotechnologique optimal d'extraction des colorants des graines de Bixa orellana; déterminer le profil chimique des colorants extraits des graines de Bixa orellana, évaluer les propriétés fonctionnelles et technologiques des colorants extraits et déterminer le potentiel à la domestication de Bixa orellana pour une production et une exploitation à grande échelle visant à en faire une filière.

\section{CONFLIT D'INTÉRÊTS}

Tous les auteurs déclarent qu'il n'y a aucun conflit d'intérêts pour la publication de cet article.

\section{CONTRIBUTIONS DES AUTEURS}

EA a contribué à ce projet d'article à travers la proposition d'un plan de rédaction, la recherche documentaire et la rédaction proprement dite. MEB a apporté sa contribution à ce projet d'article à travers ses analyses et orientations et son appui technique pour la qualité scientifique du projet d'article dans le respect des règles de publication. $\mathrm{CKG}$ a apporté sa contribution à ce projet d'article par ses lectures et recommandations et surtout ses propositions de pistes en vue de l'amélioration de sa qualité. $\mathrm{HH}$ et $\mathrm{AD}$ ont apporté leurs contributions à travers les lectures et de propositions pour son amélioration. PA a contribué en tant que coordonnateur des différentes activités liées à ce projet d'article.

\section{REMERCIEMENTS}

Les auteurs remercient les reviewers pour leurs différentes contributions à l'amélioration de la qualité de ce manuscrit.

\section{REFERENCES}

Agner AR, Bazo AP, Ribeiro LR, Salvadori DMF. 2005. DNA damage and aberrant crypt foci as putative biomarkers to evaluate the chemopreventive effect of annatto (Bixa orellana L.) in rat colon carcinogenesis. Mutat. Res., 582: 146154.

Akoègninou A, Van der Burg WJ, Van der Measen LJG, Adjakidjè V, Sinsin B, Yédomonhan H. 2006. Flore Analytique $d u$ Bénin. Backhuys Publishers.

Alexandre D-Y. 2002. Initiation à L'agroforesterie en Zone Sahélienne. Les Arbres des Champs du Plateau Central au Burkina Faso. IRD Éditions et KARTHALA.

Almeida CR, Silva RB, Marques MJ, Chavasco JK. 2012. "Evaluation of antiparasitic activity of hydroethanolic extractsfrom root, stem and leaf of Bixa orellana $L$. on Leishmania amazonensis samples," Revista da Universidade Vale do Rio Verde, 10(2): 384-391.

Alonso JR. 1998. Tratado de Fitomedicina: Bases Clínicas e Farmacológicas. ISIS Ediciones SRL, Buenos Aires: Argentina.

Anselmo GCS, Mata MERMC, Rodrigues E. 2008. Comportamento Higroscópico do 
extratoseco de urucum (Bixa orellana L.). Ciência Agrotecnica, 32(6): 18881892.

DOI:

http://dx.doi.org/10.1590/S1413-

70542008000600030

Aparnathi KD, Lata R, Sharma RS. 1990. Annatto (Bixa orellanaL.) its cultivation, preparation and usage. Int. J. Trop. Agric., 8: 80-88.

Arzour A, Belbacha K. 2015. Le risque toxicologique des colorants alimentaires. Mémoire de Master, Université des Frères Mentouri Constantine, Rép. Algérienne démocratique et populaire, $138 \mathrm{p}$.

Awika JM, Rooney LW, Waniska RD. 2004. Properties of 3-Deoxyanthocyanins from sorghum. J. Agric. Food Chem., 52: 4388-4394.

Bao J, Cai YM, Sun M, Wang G, Corke H. 2005. Anthocyanins, flavonols, and free radical scavenging activity of Chinese bayberry (Myricarubra) extracts and their color properties and stability. J. Agric. Food Chem., 53: 2327-2332.

Barbosa-Filho JM, Silva-Filho RN, Lira BF. 1988. "Teor de bixina emquatro variedades de Bixa Orellana L. cultivadas na Paraíba. Revista Brasileira de Farmacognosia, 7(8): 41-47.

Bastos ARR, Carvalho JG, Assis RP, Filho ABC. 1999. Marcha de absorcàode nutrientes emurucum (Bixa Orellana L.) tipo cultivado piavevermelhaemfase de viveiro. Cerne, 5: 76-85.

Ben Mansour H, Boughzala O, Dridi D, Barillier D, Chekir-Ghedira L, Mosrati R. 2011. Les colorants textiles sources de contamination de l'eau : CRIBLAGE de la toxicité et des méthodes de traitement. Revue des sciences de l'eau / J. Water Sci., 24(3): 209-238. DOI: http://dox.doi.org.10.7202/1006453ar

Berset C, Marty C. 1986. Potential use of annatto in extrusion cooking. Lebensmittel-Wissenschaftund-

Technologie, 19(2): 126-131.

Biloso A, Lejoly J. 2006. Etude de l'exploitation et du marché des produits forestiers non ligneux à Kinshasa. Tropicultura, 24(3): 183-188.

Birr J, Montavon S. 2004. Les additifs alimentaires: les colorants jaunes. Licence SIAL «Sécurité des Aliments », Université Paris XII Val de Marne, 31p.

Braide W, Akobundu C, Nwaoguikpe RN, Njiribaeko LC. 2011. The use of extracts from four local Nigerian plants for the staining of selected bacteria and moulds. Afr. J. Micr. R., 5(1): 79-86. DOI: http://dox.doi.org.10.5897/AJMR10.906

Bressani R, de Barneon FP-E, Braham JE, Elias LG, Gomez-Brenes R. 1983. Chemical composition, amino acid content and nutritive value of the protein of the annatto seed (Bixaorallena $L$ ). Arch. Latinoam. Nutr., 33(2): 356-376.

Cardon D, Cabalion P, Blanc B, BoulangerPenduff M, Hnawia E, Nowik W, Waikedre J, Sam C. 2010. Plantes tinctoriales des traditions et sociétés du Pacifique : de la sauvegarde des savoirs aux nouvelles perspectives d'application. Dossier spécial : Nouvelle-Calédonie et Polynésie française. Ethnopharmacologia, (46).

Cardon D, du Chatenet G. 1990. Guide des Teintures Naturelles. Neuchâtel, Delachaux et Niestlé.

Carvalho PRN, Carvalho CRL, Mantovani DMB. 1991. Estudo da composição de sementes, cachopas, folhas e galhos do urucuzeiro. In Seminário Internacional de CorantesNaturais para alimento, Campinas. Campinas: Italia.

Da Costa CLS, Chaves MH. 2005. Extração de pigmentos das sementes de Bixa Orellana L.: uma alternativa para disciplinas experimentais de química orgânica. Quim. Nova., 28(1): 149-152.

Da Silva MG, de Carvalho PR, da Rocha Tavares PE, Carvalho PRN, Kátia MVABC, Rita de Cássia SCO. 2009. Potential of Annatto in Agroindustries and Animal Feed: Fragrance, Flavor, Taste and Color of Bixa Orellana L. 
Derivatives. J. A. Sci. R., 5(12): 24822488.

De Araújo Vilar D, de Araujo Vilar MS, de Lima e Moura TFA, Raffin FN, Oliveira MR, de Oliveira Franco CF, de AthaydeFilho PF, Formiga Melo Diniz MF, Barbosa-Filho JM. Traditional Uses, Chemical Constituents, and Biological Activities of Bixa orellana L.: A Review. Sci. World. J., 11p. DOI: http://dx.doi.org/10.1155/2014/857292.

Del Prado GLG, Hernández MEG, Castillo MQ, Hernández NG, Puig JF. 2009. Bixa orellana L: a potential substance for detection of dentobacterial plaque. Rev. Cubana Estomatol. Spanish, 46(2): 1-11.

Déléké KIKE, Djego J, Gbénou J, Hounzangbé-Adoté SM, Sinsin B. 2011. Etude phytochimique des principales plantes galactogènes et emménagogues utilisées dans les terroirs riverains de la Zone cynégétique de la Pendjari. Int. J. Biol. Chem. Sci., 5(2): 618-633. DOI: 10.4314/ijbcs.v5i2.72127

Dike IP, Ibojo OO, Daramola FY, Omonhinmin AC. 2016. Phytochemical and Proximate Analysis of Foliage and Seed of Bixa Orellana Linn. Int. J. Pharm. Sci. Rev. Res., 36(2): 247-251.

Downham A, Collins P. 2000. Colouring our foods in the last and next millennium. $J$. Food Sci. Tech., 35: 5-22.

Fagbohoun L. 2014. Etude chimique de colorants naturels et matériaux résineux traditionnels du Bénin dans le domaine artisanal. Thèse de doctorat de l'université d'Abomey-Calavi (Bénin) et de l'université d'Avignon et des pays de Vaucluse (France), Spécialité: Chimie organique et substances naturelles, 295 p.

FAO. 1995. Natural colorants and dyes tuffs. Non-wood forest products series. Vol. 4.

Feldman KS, Sahasrabudhe K, Smith RS, Scheuchenzuber WJ. 1999. Immunostimulation by plant polyphenols. A relationship between tumor necrosis factor-alpha production and tannin structure. Biorganic and Medical Chemistry Letters, 9(7): 985990.

Ferreira JM, Sousa DF, Dantas MB. 2013. Effects of Bixa orellana $L$. seeds on hyperlipidemia. Phytotherapy Research, 27(1): 144-147.

Ferreira VLP, Teixeira-Neto RO, de Moura SCSR, Silva MS. 1999. Kinetics of colour degradation of water soluble commercial annatto solutions under thermal treatments. Ciencia-eTechnologia-de-Alimentos, 19(1): 37-42.

Ferreira WA, Falesi IC. 1989. Características nutricionais do fruto $e$ teor de bixinaemurucum (Bixa Orellana L.). Belém: CPATU.

Fleischer TC, Ameade EP, Mensah ML, Sawer IK. 2003. Antimicrobial activity of the leaves and seeds of Bixa orellana. Fitoterapia, 74(1-2):136-138.

Foodnet. 2002. Annatto seed. Market Information. Foodnet. www.foodnet.cgiar.org/market/Tropcom m/part2ab.htm Foodnet Market information.

Freixa B, Vila R, Vargas L, Lozano N, Adzet T, Canigueral S. 1998. Screening for antifungal activity of nineteen LatinAmerican plants. Phytotherapy Research, 12(6): 427-430.

Garcia GH, Campos R, de Torres RA. 1990. "Antiherpetic activity of some Argentine medicinal plants," Fitoterapia, 61(6): 542-546,

Gbenou JD, Ahounou JF, Ladouni P, Agbodjogbe WKDD, Tossou R, Dansou P, Moudachirou M. 2011. Propriétés anti-inflammatoires des extraits aqueux de Sterculia setigera Delile et du mélange Aframomum melegueta $\mathrm{K}$. Schum - Citrus aurantifolia Christm et Panzer. Int. J. Biol. Chem. Sci., 5(2): 634-641. DOI: 10.4314/ijbcs.v5i2.72128

Giorgi A, De Marinis P, Granelli G, Chiesa LM, Panseri S. 2013. Secondary Metabolite Profile, Antioxidant Capacity, and Mosquito Repellent 
Activity of Bixa orellanafrom Brazilian Amazon Region. J. Chem., 2013:1-10. DOI:

http://dx.doi.org/10.1155/2013/409826.

Giuliano G, Rosati C, Bramley PM. 2003. To dye or not to dye: biochemistry of annatto unveiled. Trends in Biotechnol, 21(12): 513-516. DOI: http://dx.doi.org/10.1016/j.tibtech.2003.1 0.001 .

Giusti M, Wrolstad R, Gloria M. 2001. Anthocyanins from banana bracts as potential food colorants. Food Chem., 73: 327-332.

Glew HR, Dorthy J, Vanglerajagu L, Cassius EL, Griuetti GC, Smith AP, Mark M. 1997. Amino acid and mineral composition of 24 indigenous plants of Burkano-Faso. J. Food Comp. Anal., 10: 205-217.

Gloria MBA; Vale SR, Bobbio PA. 1995. Effect of water activity on the stability of bixin in annatto extract-microcrystalline cellulose model system. Food Chem., 52: 389-391.

Guedje MN, Ntungwen Fokunang C, Tafokou Jiofack RB, Fogou Dongmo R. 2010. Opportunités d'une exploitation soutenue des plantes médicinales dans l'aménagement forestier. Int. J. Biol. Chem. Sci., 4(4): 1346-1372. DOI : http://dx.doi.org/10.4314/ijbcs.v4i4.6307 0

Guedje MN. 2004. Exploitation des produits forestiers non-ligneux (PFNL): Le cas de Garcinia lucidaVesque dans la région de BIPINDI - LOLODORF - AKOM II (Sud Cameroun).

Irobi ON, Moo-Young M, Anderson WA. 1996. Antimicrobial activity of annatto (Bixa orellana) extract. Int. J. Pharm., 34(2): $87-90$

Jansen PCM. 2005. Bixa orellana L. In PROTA (Plant Resources of Tropical Africa/Ressources végétales de l'Afrique tropicale), Jansen PCM, Cardon D (Ed). Wageningen: Netherlands.

Kapoor VP, Khan PSH, Farooqi MIH. 1975.
Chemical analysis of seeds. Part III. From 40 non-leguminous species. Sci. Cult., 41: 336-340.

Karen S. 2014. Annatto and Color Removal. Center for Dairy Research: Wisconsin.

Koffi YJJ. 2015. Une nouvelle culture dans le nord-est de la Côte d'Ivoire : le roucou (Bixa orellana) dans le département de Tanda. Germivoire. http://germivoire.net/?ivoire=detailart\&i dart $=417 \&$ rub $=152$.

Kusdiyantini E. 1998. Contribution à l'étude de la croissance et de la production d'Astaxanthine chez phaffia rhodozyma pr 190. Thèse de doctorat, Toulouse, INSA, 192p.

Levy LW, Rivandeneira DM. 2000. Annatto. In Natural Food Colorants Science and Technology, Lauro GJ, Francis FJ (eds). Marcel Dekker: New York; 117-152.

Ligia Alves DCC. 2008. Identification de facteurs opératoires influents en vue d'une production microbienne optimale de Torularhodine et de sa fonctionnalisation enzymatique, à partir d'études cinétiques. Thèse INPL, Nancy, 200p.

Lim TK. 2011. Bixa orellana. In Edible Medicinal and Non-Medicinal Plants. Springer: Dordrecht, Netherlands; 515526.

Lopes MV, Desoti VC, Caleare ADO, UedaNakamura T, Silva SO, Nakamura CV. 2012. Mitochondria superoxide anion production contributes to geranyl geraniol-induced death in leishmania amazonensis. Evidence-Based Complementary and Alternative Medicine, ID298320: 9p. DOI: $10.1155 / 2012 / 298320$

Lorenzi H, Matos FJA. 2002. Plantas medicinais no Brasil: nativas e exóticas. Nova Odessa: Instituto Plantarum.

Madiélé AB, Quio Zhao JM, Thiery V, Agnaniet $\mathrm{H}$, Brunet $\mathrm{C}$, Graber $\mathrm{M}$, Ouamba JM. 2015. Caractérisations analytiques des extraits colorants des plantes tinctoriales d'Afrique Centrale. 
Lebanese Sci. J., 16(1):33-33.

Maga JA, Kim CH. 1990. Stability of natural colourants (Annatto, beet, paprica and turmeric) during extrusion cooking. Lebensmittel-Wissenschaft undTechnologie, 23(5):427-432.

Magda TR. 2003. Going back to natural red colors (red sorghum, cochineal, and Monascus). Drink Technology and Market, 7 : 1433-1594.

Mara. 1999. L'agriculture ivoirienne à l'aube du 21ème siècle. Rapport interministériel des Ministères de l'Agriculture et des Ressources Animales, de l'Environnement et de la forêt, de l'enseignement Supérieur et de la Recherche scientifique, Abidjan, Côte d'Ivoire, P. 243.

Metta O, Arunsri J, Chanapong R. 2009. Antibacterial effect of crude alcoholic and aqueous extracts of six medicinal plants against Staphylococcus aureus and Escherichia coli. J Health Res., 23(3): 153-156.

Moraes SA. 2007. Subprodutos da agroindústria e indicadores externos de digestibilidade aparente em caprinos. Tese de doctorado, Universidad Federal de Minas Gerais, Belo Horizonte.

OCDE. 2002. L'économie locale de Bondoukou: comptes, acteurs et dynamisme de l'économie locale. Rapport de l'Organisation de Coopération et de Développement Economique (OCDE) SAH/D521/2001, Paris, France, P.104.

Omonhinmin AC, Dike IP, Agbara U. 2013. In vivo antioxidant assessment of two antimalarial plants- Allamamda cathartica and Bixa Orellana. Asian Pacific J.Tropi Biomed., 3(5): 388-394.

Parimalan R, Giridhar P, Rajasekaran T, Ravishankar GA. 2007. Annatto fruit pericarp: newer source as a potential fuel. Energy Fuel, 21(2): 1181-1182.

Pedrosa JP, Cirne LEMR, Magalhães NJM. 1999. Teores de bixina e proteína emsementes de urucumemfunção do tipo e do período de armazenagem. Revista Brasileira de Engenharia Agrícola e Ambiental., 1(1): 121-123.

Pereira ES, Regadas Filho JGL, Freitas ER, Neiva JNM, Cândido MJD. 2009. Valor energético de subprodutos da agroindústria brasileira. Archivos de Zootecnia, 58(223): 455-458. DOI: http://dx.doi.org/10.4321/S000405922009000300015.

Periyasamy L, Kumar Y. 2016. GC-MS analysis and in-vitro cytotoxic studies of Bixa orellana seed extract against cancer cell line. International Journal of Pharmacy and Pharmaceutical Sciences, 8(1): 1024-1036.

Pigeaud R. 2003. Préhistoire de la couleur. In Archéologia $\mathrm{n}^{\circ}$ 406. 18-23.

Prabhakara Rao PG, Jyothirmayi T, Balaswamy K, Satyanarayana A, Rao DG. 2004. Effect of processing conditions on the stabilityof annatto (Bixa orellana L.) dye incorporated into some foods. LWT - Food Science and Technology, 38(7): 779-784. DOI: http://dx.doi./10.1016/j.lwt.2004.08.015

Prathima D, Sujitha A, Usha R. 2016. Phytochemical Screening and Antimicrobial Activity of Bixa Orellana Linn. IJPPR., 8(7): 1078-1082.

PROVAPEG. 2015. Rapport narratif final. Projet de Valorisation des Produits du Petit Elevage et de Gestion des Parcours Agropastoraux dans le Gorgol (PROVAPEG), Période du 2 janvier 2012 au 30 Juin 2015. PROVAPEG.

Rajendra SB. 2014. Antibacterial activity of the Ethanolic leaves extract of Bixa orellana. World J. Pharm. Pharm. Sci., 3(9): 389-394.

Rajib A, Km Monirul I, Musaddik A, Haque E. 2009. Hepatoprotective Activity of Methanol Extract of Some Medicinal Plants Against Carbon Tetrachloride Induced Hepatotoxicity in Albino Rats. Global J. Pharmacol., 3(3): 116-122.

Renata R-M, Rosa ME-GM, Erick B-G, Marina V-K, Huges H. 2006. 
Preliminary studies toward genetic improvement of annatto (Bixa orellana L.). Scientia Horticulturae, 109: 165172.

DOI:

http://dx.doi.org.10.1016/j.scienta.2006. 03.011 .

Rios AO, Antunes LMG, Bianchi MLP. 2009. Protecão de carotenóides contra radicais libres gerados no tratamento de câncercom cisplatina. Rev. Nutr., 20: 343-350.

Rodriguez-amaya DB. 2008. Updated Brazilian database on food carotenoids: Factors affecting carotenoid composition. J. Food Com. Anal., 21(6): 445-463.

Sangvikar S, Malgaonkar M, Sharma C, Kumar S, Murthy SN. 2015. Comparative phytochemical screening of qualitative and quantitative parameters of Bixa orellana L. World J. Pharm Pharm Sci., 4(12): 1001-1017.

Satyanarayana A, PrabhakaraRao PG, Rao DG. 2003. Chemistry processing and toxicology of annatto (Bixa orellana $\mathrm{L}$ ). J. Food Sci. Technol., 40(2): 131-141.

Senthil KP, Reddy YR, Ramesh S, Gobinath S and Ramana DBV. 2007. Evaluation of pigment extracted annatto seed (Bixa orellana) by chemical, in-vitro and insaccotechniques in buffaloes. Buffalo Bulletin, 26(1): 5-9.

Sessou P, Farougou S, Azokpota P, Youssao I, Yehouenou B, Ahounou S, Codjo Koko Sohounhloue D. 2013. Inventaire et analyse des pratiques endogènes de conservation du Wagashi, un fromage traditionnel produit au Bénin. Int. J. Biol. Chem. Sci., 7(3): 938-952. DOI: http://dx.doi.org.10.4314/ijbcs.v7i3.5

Silva SNS, Amaral CLF, Rebouças TNH. 2010. Adoption of conservation practices on farm and selection of varieties by producers of annatto in the city of Vitoria da Conquista-BA. Revista Brasileira de Agroecologica, 5: 106113.

Sinha K, Saha PD, Datta S. 2012 (a).
Response surface optimization and artificial neural network modeling of microwave assisted natural dye extraction from pomegranate rind. Industrial Crops and Products, 37: 408414.

Sinha K, Saha PD, Datta S. 2012 (b). Extraction of natural dye from petals of Flame of forest (Buteamonosperma) flower: process optimization using Response Surface Methodology (RSM). Dyes and Pigments, 94: 212-216.

Sivakumar V, Vijaeeswarri J, Anna JL. 2011. Effective natural dye extraction from different plant materials using ultrasound. Industrial Crops and Products, 33: 116-122.

Soumya V, Venkatesh P, Hari PK, Shrishailappa B. 2010.Microwave facilitated extraction of Bixin from Bixa orellana and its in-vitro antioxidant activity. Der Pharmacia Lettre, 2(2): 479-485

Taham T, Cabral FA, Barrozo MAS. 2015. Extraction of bixin from annatto seeds using combined technologies. $J$. Supercrit. Fluids, 100: 175-183.

Thomas E, Colvin PM, Rosen SB, Zuccarini C, Petzer S. 2005. HIV prevalence study and costing analysis undertaken for the development of an HIV/AIDS workplace strategy for buffalo city municipality. Tech. Rep. Medical Research Council: Buffalo, NY, USA.

Ul-Islam S, Rather LJ, Mohammad F. 2016. Phytochemistry, biological activities and potential of annatto in natural colorant production for industrial applications A review. J. Adv. Res., 7:499-514. DOI: http://dx.doi.org/10.1016/j.jare.2015.11. 002

Utiyama CE, Miyada VS, Figueiredo AN, Oetting L. 2002. Digestibilidade de nutrientes do resíduo de semente processadas de urucum (Bixa orellana) para suinos. In Reunião Anual da Sociedade Brasileira de Zootecnia, Recife. Recife: Sociedade Brasileira de 
Zootecnia.

Valdés AFC, Martínez JM, Rodríguez DA, Lizama RS, Gaitén YG. 2011. Actividad antimalaria de un extracto hidroalcohólico de Bixa Orellana $L$. Revista Cubana de MedicinaTropical, 63: $181-185$.

Valentin SA, Melo JO, Pinho RJ, Schimidt G, Pereira FJ, Cardoso MLC. 2013. Antilipemic activity of ethanolic and hexane extracts from seeds of Bixa orellana Linn. In hyperlipidemic rats. $J$. Med. Pl. Res., 7(4):165-169.

Valério MA, Lima R, Maria I, Braga N, José A; Rodrigues M, Maria L. 2015. Annatto seed residue (Bixa orellana L.): nutritional quality. Food Sci. Technol, Campinas, 35(2): 326-330. DOI: http://dx.doi.org/10.1590/1678457X.6539

Van den Berg H, Faulks R, Granado HF, Hirschberg J, Olmedilla B, Sandmann G, Southon S, Stahl W. 2000. The potential for the improvement of carotenoid levels in foods and likely systemic effects. $J$. Sci. Food Agric., 80: 880-912.

Venugopalan A, Giridhar P, Ravishankar GA.
2011. Food, ethnobotanical and diversified applications of Bixa Orellana L.: a scope for its improvement through biotechnological mediation. Ind. J. Fund. App. Life Sci., 1: 9-31.

Wurts ML, Torreblanca RA. 1983. Analysis of the seed, L. (annatto) and the waste generated in the extraction of its pigments. Arch. Latinoam Nutr., 33: 606-619.

Yolmeh M, Habibi-Najafi MB, Farhoosh R. 2014. Optimisation of ultrasoundassisted extraction of natural pigment from annatto seeds by response surface methodology (RSM). Food Chem., 155: 319-324.

Zarza-García AL, Sauri-Duch E, RaddatzMota D, Cuevas-Glory LF, PinzónLópez LL, Rivera-Cabrera F, MendozaEspinoza JA. 2017. Pharmacological, phytochemical and morphological study of three Mayan accessions of Bixa orellana L. leaves. Emir. J. Food Agric., 29(3): 163-169. DOI: http://dox.doi/10.9755/ejfa.2016-091283. 